\title{
HS 1857+5144: a hot and young pre-cataclysmic variable
}

\author{
A. Aungwerojwit ${ }^{1,2}$, B. T. Gänsicke ${ }^{1}$, P. Rodríguez-Gil ${ }^{3}$, H.-J. Hagen ${ }^{4}$, O. Giannakis $^{5}$, C. Papadimitriou ${ }^{5}$, \\ C. Allende Prieto ${ }^{6}$, and D. Engels ${ }^{4}$ \\ 1 Department of Physics, University of Warwick, Coventry, CV4 7AL, UK \\ e-mail: A. Aungwerojwit@warwick.ac.uk \\ 2 Department of Physics, Faculty of Science, Naresuan University, Phitsanulok 65000, Thailand \\ Instituto de Astrofísica de Canarias, 38200 La Laguna, Tenerife, Spain \\ ${ }^{4}$ Hamburger Sternwarte, Universität Hamburg, Gojenbergsweg 112, 21029 Hamburg, Germany \\ Institute of Astronomy and Astrophysics, National Observatory of Athens, PO Box 20048, Athens 11810, Greece \\ ${ }^{6}$ McDonald Observatory and Department of Astronomy, University of Texas, Austin, TX 78712, USA
}

Received 10 February 2007 / Accepted 11 April 2007

\begin{abstract}
Aims. We report the discovery of a new white dwarf/M dwarf binary, HS 1857+5144, identified in the Hamburg Quasar Survey (HQS). Methods. Time-resolved optical spectroscopy and photometry were carried out to determine the properties of this new cataclysmic variable progenitor (pre-CV).

Results. The light curves of HS $1857+5144$ display a sinusoidal variation with a period of $P_{\text {orb }}=383.52$ min and peak-to-peak amplitudes of $0.7 \mathrm{mag}$ and $1.1 \mathrm{mag}$ in the $B$-band and $R$-band, respectively. The large amplitude of the brightness variation results from a reflection effect on the heated inner hemisphere of the companion star, suggesting a very high temperature of the white dwarf. Our radial velocity study confirms the photometric period as the orbital period of the system. A model atmosphere fit to the spectrum of the white dwarf obtained at minimum light provides limits to its mass and temperature of $M_{\mathrm{wd}} \simeq 0.6-1.0 M_{\odot}$ and $T_{\mathrm{wd}} \simeq 70000-100000 \mathrm{~K}$, respectively. The detection of He II $\lambda 4686$ absorption classifies the primary star of HS $1857+5144$ as a DAO white dwarf. Combining the results from our spectroscopy and photometry, we estimate the mass of the companion star and the binary inclination to be $M_{\mathrm{sec}} \simeq 0.15-0.30 M_{\odot}$ and $i \simeq 45^{\circ}-55^{\circ}$, respectively.

Conclusions. We classify HS $1857+5144$ as one of the youngest pre-CV known to date. The cooling age of the white dwarf suggests that the present system has just emerged from a common envelope phase $\sim 10^{5} \mathrm{yr}$ ago. HS $1857+5144$ will start mass transfer within or below the $2-3 \mathrm{~h}$ period gap.
\end{abstract}

Key words. stars: binaries: close - stars: individual: HS 1857+5144

\section{Introduction}

Post-common envelope binaries (PCEBs), i.e. detached white dwarf-main sequence binaries, originate from wide binaries comprising unequal main sequence components. Once the more massive star evolves through the giant phase and fills its Roche lobe, unstable mass transfer onto the unevolved star starts. The high accretion rate subsequently brings the system into a common envelope phase (CE). Friction between the stellar components and the envelope shrinks the binary orbital separation and eventually ejects the envelope from the system, resulting in the majority of PCEBs having orbital periods of a few days (Willems $\&$ Kolb 2004). The binary separations of PCEBs are believed to be further reduced through angular momentum loss via magnetic braking and/or gravitational radiation. The main-sequence star will ultimately fill its Roche lobe and start mass transfer onto the white dwarf, which turns the binary into a cataclysmic variable (CV). While this general scenario is widely accepted, the details of the evolution through the common envelope phase, as well as the subsequent orbital angular momentum loss are poorly understood. Consequently predictions made by binary population models are rather uncertain.

As PCEBs are simple objects in terms of their stellar components, they offer a large potential in constraining and calibrating the physics of both CE evolution and orbital angular momentum loss. This has implications for a wide range of astronomical objects such as SN Ia progenitors, X-ray binaries, or neutron star binaries as likely progenitors of short gamma-ray bursts. However, until recently PCEBs received little observational attention, largely due to the lack of a dedicated search for such systems. Schreiber \& Gänsicke (2003) analysed the properties of 30 well-studied PCEBs and showed that the known population of these systems is extremely biased towards young systems consisting of hot white dwarfs and late type companions, as the majority of the known PCEBs were initially selected as blue objects. A substantial improvement in the statistics of PCEB properties will be possible through the exploitation of large extragalactic surveys such as the Hamburg Quasar Survey (HQS, Hagen et al. 1995) or the Sloan Digital Sky Survey (SDSS, York et al. 2000).

Here we present a detailed follow-up study of the new PCEB HS $1857+5144$, which has been discovered in our ongoing effort to identify CVs and pre-CVs in the HQS (see Gänsicke et al. 2002; Aungwerojwit et al. 2005 for details on the project). In Sect. 2 we describe the observations and data reduction. The orbital ephemeris of HS $1857+5144$ is determined in Sect. 3. In Sects. 4-5 we analyse the nature of the stellar components and discuss the future evolution of the system. 


\section{Observations and data reduction}

\subsection{Spectroscopy}

An identification spectrum of HS $1857+5144$ was obtained in August 1992 with the Boller \& Chivens Cassegrain spectrograph on the 2.2-m telescope at Calar Alto Observatory. The spectrum is characterised by a blue continuum superimposed by strong Balmer emission lines. Subsequent time-series, intermediate resolution spectroscopy of HS $1857+5144$ was performed in July 2004 at the 2.7-m Harlan J. Smith telescope at McDonald Observatory equipped with the Large Cassegrain Spectrograph (LCS), covering $\sim 10 \mathrm{~h}$ (35 spectra) in total. The individual spectra were obtained through a 1 "slit and grating \#43 and imaged on the $800 \times 800$ pixel TI1 CCD camera. This setup provided access to the $\lambda \lambda 3670-5050$ wavelength range at $3.5 \AA$ spectral resolution. The reduction and optimal extraction of the spectra were performed using standard long-slit spectroscopy packages within IRAF ${ }^{1}$. Four additional high resolution spectra were obtained using the Intermediate Dispersion Spectrograph and Imaging System (ISIS) on the 4.2-m William Herschel Telescope (WHT) on La Palma in July 2006, covering the orbital minimum, maximum, and the quadrature phases. The blue arm of the spectrograph was equipped with the R1200B grating, providing a spectral resolution of $\simeq 1 \AA$ over the wavelength range 4200-5000 $\AA$. The data were reduced using the Figaro within Starlink package as well as Pamela and Molly written by T. Marsh ${ }^{2}$.

Spectroscopic characteristics. Figures 2 and 3 illustrate the orbital phase-dependent variation of the emission line profiles of HS 1857+5144 from the McDonald and WHT spectra, respectively. The emission lines greatly vary in strength over the orbital cycle, with maximum line fluxes occurring at $\varphi \simeq 0.5$ and disappearing around $\varphi \simeq 0.0$. The dominant lines detected in the McDonald spectra belong to the Balmer series, but the high quality of the WHT spectra reveals a multitude of narrow emission lines, e.g. the $\mathrm{C}$ III/ $\mathrm{N}$ III $\lambda 4650$ Bowen blend, $\mathrm{Mg}$ II, $\mathrm{N}$ II, $\mathrm{O}$ II/ $\mathrm{C}$ III blend, and $\mathrm{N}$ III/Fe III blend. This emission spectrum is a characteristic of known PCEBs containing a cool secondary star irradiated by a very hot primary component, such as BE UMa (Ferguson et al. 1981; Ferguson \& James 1994), EC 11575-1845 (Chen et al. 1995), and HS 1136+6644 (Sing et al. 2004). The strengths of the Balmer emission lines vary in phase with those of $\mathrm{He}$ I, $\mathrm{C}$ III/ $\mathrm{N}$ III, indicating that all emission lines come from the same source. The WHT spectra also reveal that the Balmer emission lines have double-peaked profiles, with peak separations of $\sim 170 \mathrm{~km} \mathrm{~s}^{-1}$ for $\mathrm{H} \beta$ (Fig. 3, small window) and $\sim 150 \mathrm{~km} \mathrm{~s}^{-1}$ for $\mathrm{H} \gamma$ which are most likely caused by non-LTE effects in the strongly irradiated atmosphere of the companion star (Barman et al. 2004).

During the orbital faint phase, $\varphi \simeq 0.0$, weak absorption Balmer lines are observed in both sets of spectra, with He II $\lambda 4686$ absorption also detected in the WHT spectra. The detection of $\mathrm{He}$ II $\lambda 4686$ classifies the primary star in HS $1857+5144$ as a DAO white dwarf.

\footnotetext{
${ }^{1}$ IRAF is distributed by the National Optical Astronomy Observatories, which are operated by the Association of Universities for Research in Astronomy, Inc., under cooperative agreement with the National Science Foundation.

2 www.warwick.ac.uk/go/trmarsh
}



Fig. 1. A $10^{\prime} \times 10^{\prime}$ finding chart for HS $1857+5144$ obtained from the Digitized Sky Survey. The J2000 coordinates of the star are $\alpha=$ $18^{\mathrm{h}} 58^{\mathrm{m}} 32.12^{\mathrm{s}}$ and $\delta=+51^{\circ} 48^{\prime} 57.74^{\prime \prime}$. The comparison and check stars used in the photometry are marked by "C1"-"C4" (see Sect. 2.2 for details).

\subsection{Photometry}

We obtained a total of $\sim 60 \mathrm{~h}$ of time-series CCD photometry of HS $1857+5144$ (Fig. 1) during the period July 2003 to June 2006 (Table 1). Filterless photometry was carried out in 2003/4 using the 1-m Optical Ground Station (OGS) and the 0.82m IAC80 telescope at the Observatorio del Teide on Tenerife. Both telescopes were equipped with Thomson $1 \mathrm{k} \times 1 \mathrm{k}$ pixel CCD cameras. Photometric observations were also carried out with the 1.2-m telescope at Kryoneri Observatory and a $516 \times$ 516 pixel Photometrics SI-502 CCD camera. The OGS data were reduced in a standard fashion with IRAF, and the instrumental magnitudes of the object and comparison stars in the field were extracted using the point spread function (PSF) packages. Differential magnitudes of HS $1857+5144$ were then computed relative to the comparison star "C1" (USNO-A2.0 135010080469: $R=13.2, B=14.7$ ) whose brightness variation was found to be negligible against the check star "C2" (USNOA2.0 1350-10078502: $R=13.5, B=14.6)$. The IAC80 and Kryoneri data were reduced using the pipeline described by Gänsicke et al. (2004) which employs MIDAS for bias and dark current subtraction and flat fielding, and performs aperture photometry over all visible objects using Sextractor (Bertin \& Arnouts 1996). Differential magnitudes for HS 1857+5144 were calculated from the Kryoneri data using again the comparison star "C1" and the check star "C2". For the IAC80 data, the comparison star "C3" (USNO-A2.0 1350-10078272: $R=14.5, B=$ 16.1) and the check star "C4" (USNO-A2.0 1350-10079362: $R=16.4, B=17.8$ ) were used. Additional $B$-band and $R$-band light curves of HS $1857+5144$ were obtained at Kryoneri observatory in May/June 2006 and reduced in the same way as the filterless data from this telescope. Samples of white light, $B$-band and $R$-band light curves are displayed in Fig. 4.

Light curve morphology. The light curves of HS $1857+5144$ (Fig. 4) display a smooth quasi-sinusoidal modulation with a period of $\simeq 6.4 \mathrm{~h}$ and peak-to-peak amplitudes of $0.7 \mathrm{mag}$ in the $B$-band, $1.1 \mathrm{mag}$ in the $R$-band, and $0.9 \mathrm{mag}$ in white light. The minimum in the $B$-band light curve is nearly flat for $\simeq 0.15$ 


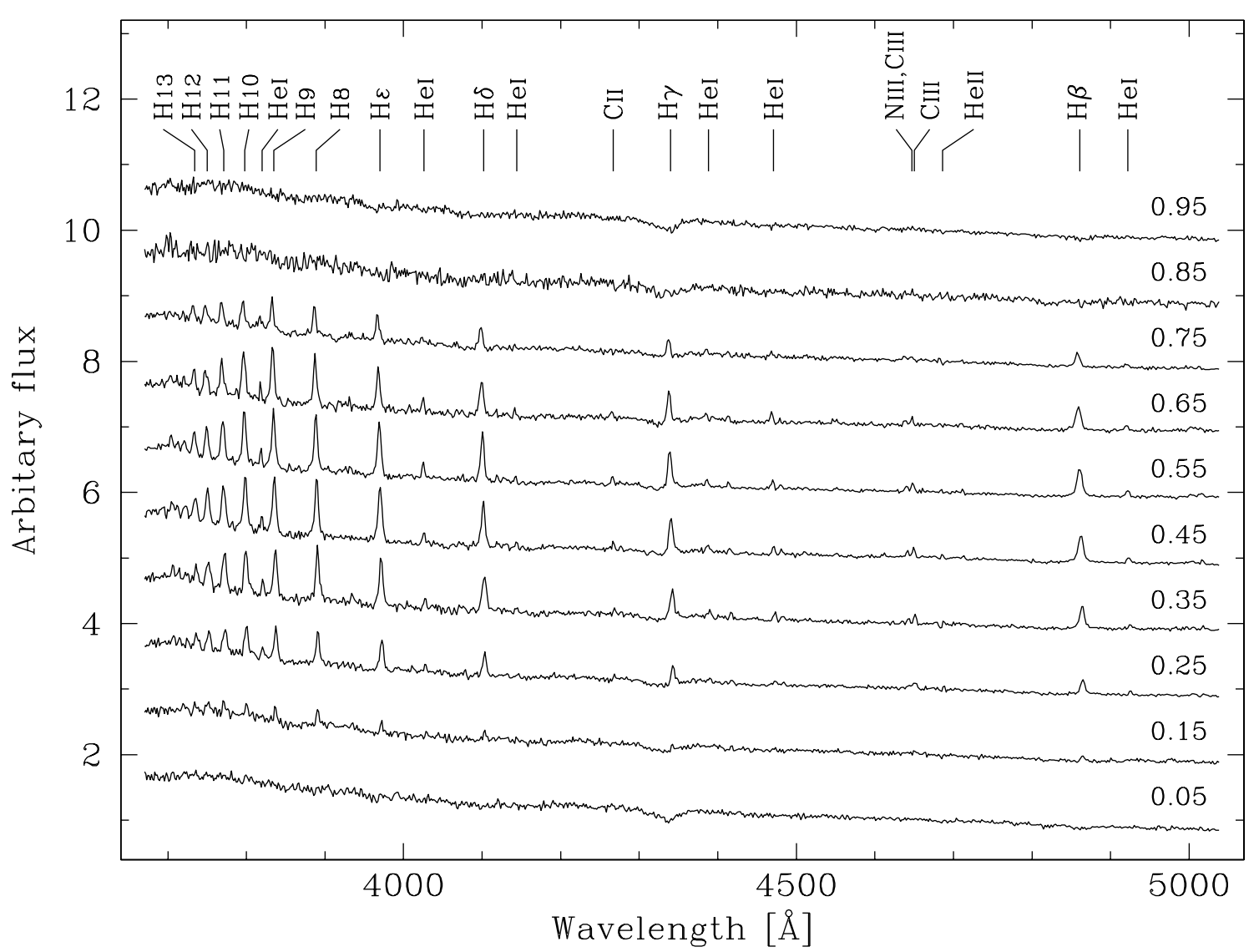

Fig. 2. Phase-binned spectra of HS 1857+5144 obtained at McDonald Observatory. These spectra show a clear modulation of the emission line strengths with orbital phase.

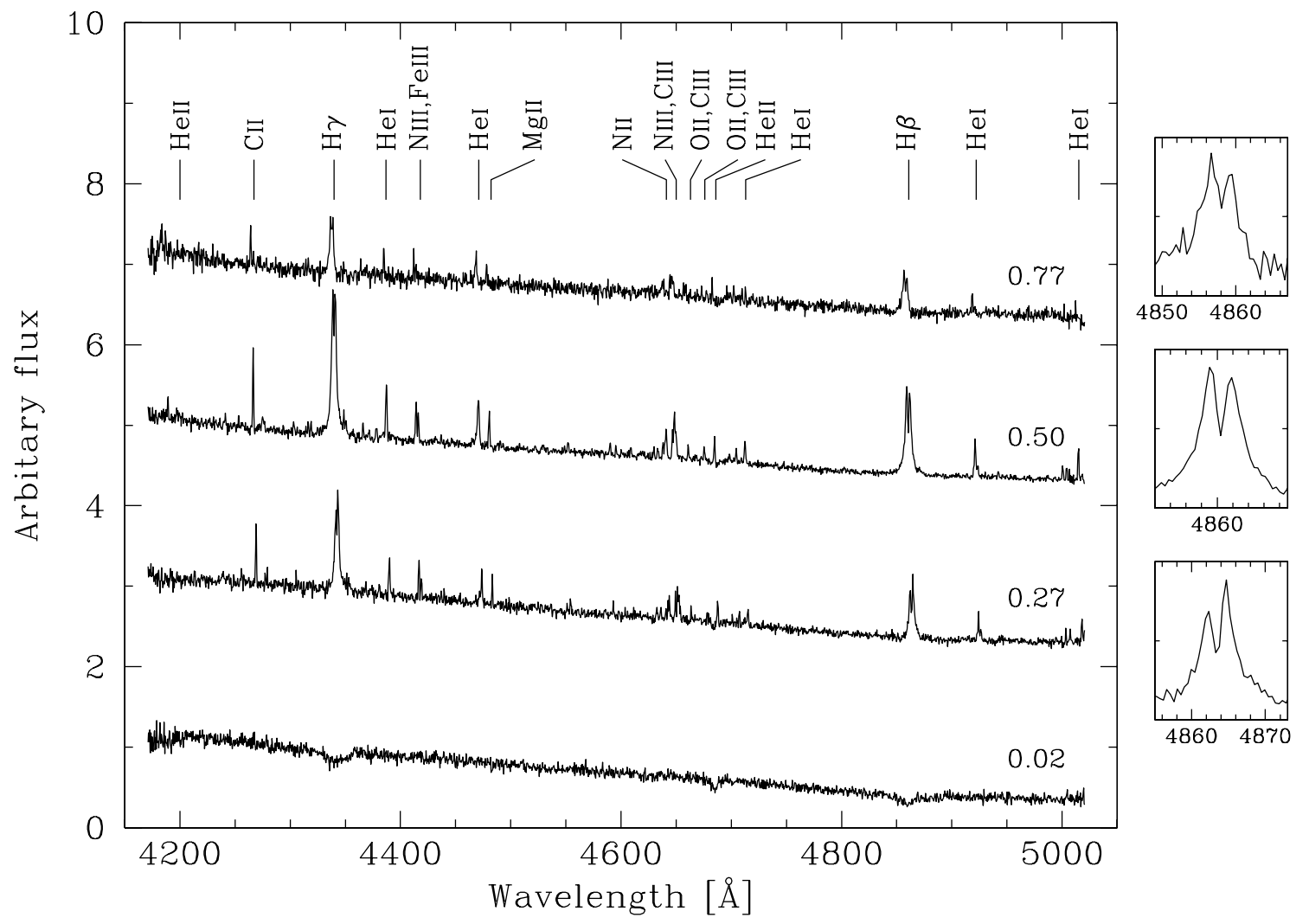

Fig. 3. Main panel: high resolution spectra of HS $1857+5144$ at different orbital phases from $0.02-0.77$ obtained at the WHT. Small windows: close up of the evolution of the $\mathrm{H} \beta$ emission line profile in the orbital phase range $0.27-0.77$ (from bottom to top). 
Table 1. Log of the observations.

\begin{tabular}{lccccc}
\hline \hline Date & UT & Telescope & $\begin{array}{c}\text { Filter/ } \\
\text { Grism }\end{array}$ & $\begin{array}{c}\text { Exp. } \\
(\mathrm{s})\end{array}$ & Frames \\
\hline 1992 Aug. 10 & $21: 51$ & CA2.2 & & 1500 & 1 \\
2003 Jul. 10 & $21: 03-01: 01$ & OGS & clear & 17 & 610 \\
2003 Jul. 13 & $21: 16-01: 00$ & OGS & clear & 12 & 751 \\
2003 Jul. 21 & $19: 15-02: 25$ & KY & clear & 30 & 671 \\
2003 Jul. 23 & $18: 56-02: 32$ & KY & clear & $20-45$ & 311 \\
2004 May 21 & $01: 43-05: 25$ & IAC80 & clear & 40 & 231 \\
2004 May 23 & $00: 52-02: 07$ & KY & clear & $20-30$ & 199 \\
2004 May 25 & $21: 34-02: 26$ & KY & clear & 20 & 587 \\
2004 May 26 & $23: 14-02: 29$ & KY & clear & 20 & 335 \\
2004 May 27 & $02: 51-05: 12$ & IAC80 & clear & 40 & 286 \\
2004 Jun. 09 & $21: 40-02: 20$ & KY & clear & 20 & 608 \\
2004 Jul. 16 & $04: 13-11: 01$ & McD & $\# 43$ & 600 & 31 \\
2004 Jul. 19 & $04: 25-08: 58$ & McD & $\# 43$ & 600 & 4 \\
2006 May 28 & $21: 21-02: 14$ & KY & $R$ & $70-80$ & 212 \\
2006 May 29 & $19: 53-02: 21$ & KY & $R$ & 80 & 267 \\
2006 May 30 & $20: 58-02: 21$ & KY & $R$ & 80 & 186 \\
2006 Jun. 04 & $20: 15-02: 14$ & KY & $B$ & $90-110$ & 196 \\
2006 Jul. 02 & $21: 17$ & WHT & R1200 & 600 & 1 \\
2006 Jul. 03 & $21: 21$ & WHT & R1200 & 600 & 1 \\
2006 Jul. 04 & $21: 17$ & WHT & R1200 & 600 & 1 \\
2006 Jul. 05 & $21: 16$ & WHT & R1200 & 600 & 1 \\
\hline
\end{tabular}

Notes. CA2.2m: 2.2-m telescope, Calar Alto Observatory; IAC80: 0.82-m telescope, Observatorio del Teide; KY: 1.2-m telescope, Kryoneri Observatory; McD: 2.7-m Harlan J. Smith Telescope, McDonald Observatory; OGS: 1-m Optical Ground Station, Observatorio del Teide; WHT: 4.2-m William Herschel Telescope, Roque de Los Muchachos Observatory.

orbital cycle, whereas the shape of the minimum in the $R$-band is rounder. No sign of the typical short-period flickering of accreting systems is detected, which classifies HS $1857+5144$ as a detached binary. The low-amplitude scatter seen in the light curves in Fig. 4 is caused by residual flat field structures and poor tracking of the Kryoneri telescope. The observed periodic brightness variation is characteristic of a large reflection effect on the heated face of the secondary star, irradiated by a hot primary star (e.g. TW Crv, Chen et al. 1995; KV Vel, Hilditch et al. 1996; and HS 2333+3927, Heber et al. 2004). Finally, HS 1857+5144 was found at a constant mean magnitude of $\simeq 16.2$ throughout our observing runs, consistent with USNO-A2.0 measurements of HS $1857+5144(R=16.3$ and $B=15.7)$.

\section{Analysis}

\subsection{Orbital period and ephemeris}

A qualitative inspection of the light curves presented in Sect. 2.2 provided an estimate of the orbital period of $\simeq 6.4 \mathrm{~h}$. In order to obtain a precise value, we shifted the magnitudes of each observing run so that their minima match that of the 2003 July 21 Kryoneri data, which covered an entire orbital cycle. We then subjected the combined 2003-2006 data to a time-series analysis within the MIDAS/TSA context using Schwarzenberg-Czerny's (1996) ORT method, a variation of the analysis-of-variance technique which fits orthogonal polynomials to the data folded over a set of trial periods. The ORT periodogram (Fig. 5) contains an unambiguous peak at $3.755 \mathrm{~d}^{-1}$. A sine fit to the combined photometric data defined the following ephemeris:

$T_{0}=\mathrm{HJD} 2452831.5475(17)+0.26633357(8) \times E$,



Fig. 4. Filterless, $R$, and $B$ (from top to bottom) sample light curves of HS $1857+5144$ obtained with the $1.2-\mathrm{m}$ telescope at Kryoneri Observatory.

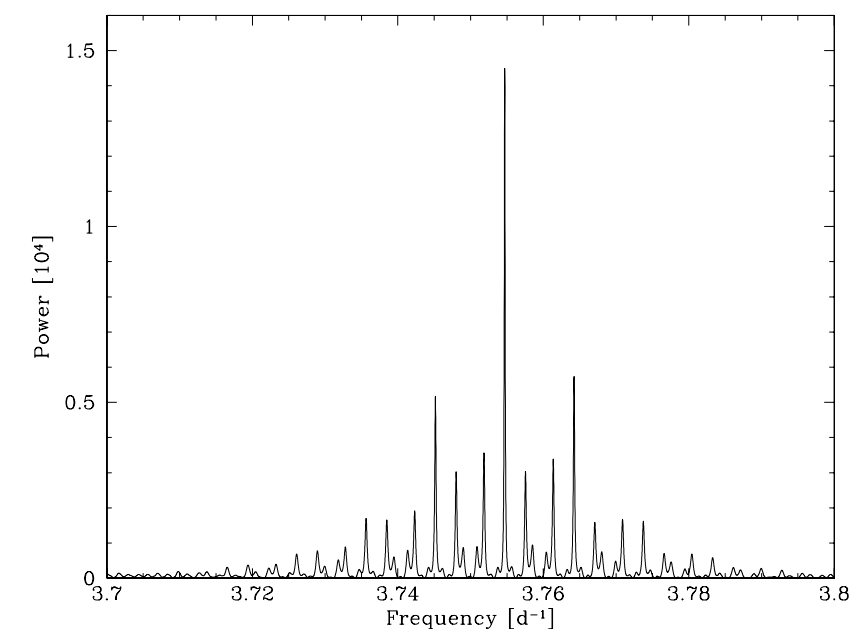

Fig. 5. The ORT periodogram of HS $1857+5144$ computed from all photometric data.

where $T_{0}$ is defined as the time of inferior conjunction of the secondary star (=orbital minimum in the light curves). We conclude that the orbital period of HS $1857+5144$ is $P_{\text {orb }}=383.5203 \pm$ 0.0001 min. Figure 6 (bottom two panels) shows the Kryoneri $B$-band and $R$-band light curves folded according to the above ephemeris.

\subsection{Radial velocities and equivalent widths}

In order to spectroscopically confirm the orbital period of HS $1857+5144$, we first measured radial velocity variations of the $\mathrm{H} \beta, \mathrm{H} \gamma, \mathrm{H} \delta, \mathrm{H} \epsilon$, and $\mathrm{H} 10$ emission lines separately by crosscorrelating the observed line profiles with a single Gaussian with a full-width at half-maximum $(F W H M)$ of $250 \mathrm{~km} \mathrm{~s}^{-1}$ for $\mathrm{H} \beta$ and $\mathrm{H} \epsilon$, and of $300 \mathrm{~km} \mathrm{~s}^{-1}$ for $\mathrm{H} \gamma, \mathrm{H} \delta$, and $\mathrm{H} 10$ within MOLLY. The ORT periodograms calculated from the radial velocities of the individual lines consistently favoured an orbital frequency of $\sim 3.75 \mathrm{~d}^{-1}$, in good agreement with the photometric result. The radial velocity amplitudes determined from the different Balmer lines varied in the range $\sim 175-215 \mathrm{~km} \mathrm{~s}^{-1}$.

In order to obtain a more robust measure of the radial velocity of the companion star in HS $1857+5144$, we determined an average radial velocity of the Balmer lines by fitting 


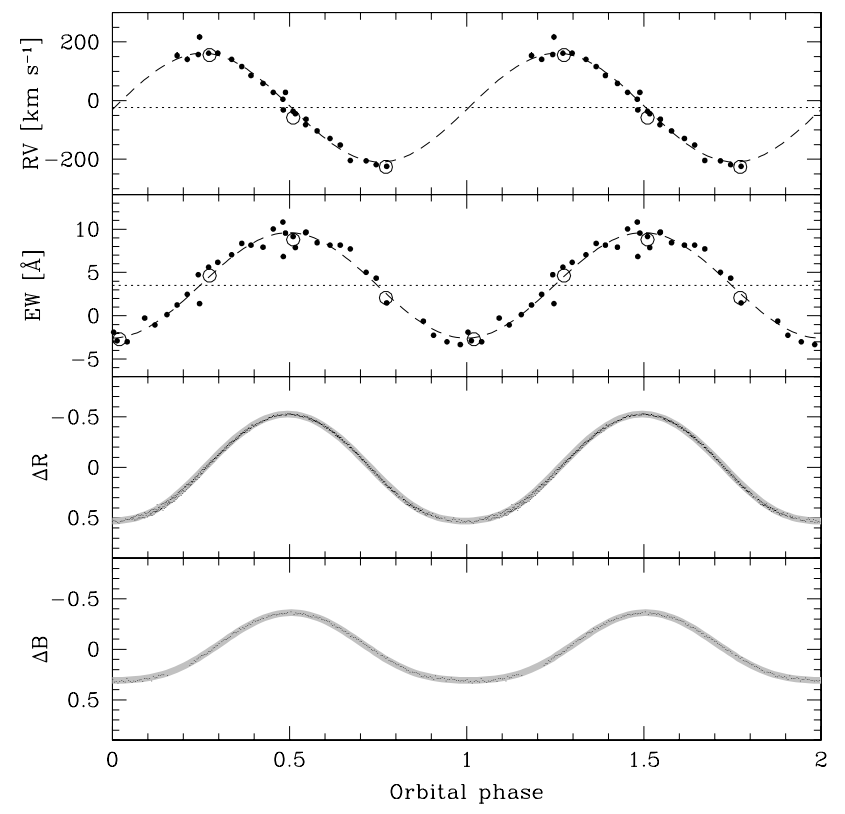

Fig. 6. Spectroscopic and photometric data of HS $1857+5144$ folded over the photometric orbital period of 383 min given in Eq. (1). Top two panels: the average of the Balmer radial velocities and $\mathrm{H} \beta$ equivalent width variations along with the best sine fit (dashed line); the filled and open circles represent the Mcdonald and WHT data, respectively. The error bars in the radial velocity measurements are included in the plot, but of similar size as the points. The uncertainties in the values of the equivalent widths are dominated by systematic effects of the order $\sim 1 \AA$. Bottom two panels: $R$-band and $B$-band light curves obtained during May/June 2006 along with the synthetic light curves (gray line) computed with the program PHOEBE for $M_{\mathrm{wd}}=0.72 M_{\odot}, M_{\mathrm{sec}}=0.21 M_{\odot}$, $i=53^{\circ}$, and $T_{\mathrm{wd}}=100000 \mathrm{~K}$. Phase zero is defined as inferior conjunction of the secondary star. Note that phase of the radial velocity curve is offset with respected to equivalent width variation curve and light curves by $\simeq 0.25$, consistent with an origin of the emission lines on the heated inner hemisphere of the secondary star (see Sect. 3.3 for details).

simultaneously the Balmer series $\mathrm{H} \beta$ to $\mathrm{H} 13$ with a set of 10 Gaussians. The wavelengths of all Gaussians were fixed to their laboratory wavelengths relative to that of $\mathrm{H} \delta$, and only the wavelength of $\mathrm{H} \delta$, as well as the widths and amplitudes of all 10 Gaussians were used as free parameters. The average Balmer line radial velocities are listed in Table 2 and are shown in Fig. 6 (top panel) folded over the ephemeris given in Eq. (1). A sine fit to the folded velocities and their errors results in an amplitude of $K_{\mathrm{em}}=185.2 \pm 4.9 \mathrm{~km} \mathrm{~s}^{-1}$ and $\gamma=-24.0 \pm 6.5 \mathrm{~km} \mathrm{~s}^{-1}$. Note that this velocity amplitude does not represent the radial velocity amplitude of the centre of mass of the secondary star, but that of the illuminated hemisphere. Since the centre of light is located closer to the centre of mass of the system than the centre of mass of the secondary star, the "true" radial velocity amplitude of the secondary star should therefore be larger than the observed velocity amplitude, $K_{\mathrm{em}}=185.2 \pm 4.9 \mathrm{~km} \mathrm{~s}^{-1}$. We will determine a $\mathrm{K}$-correction in Sect. 4.3.

We also analysed the variation of the equivalent width $(E W)$ of the $\mathrm{H} \beta$ line (Table 2). The Scargle periodogram calculated from these measurements contained two equally significant signals at $3.409 \mathrm{~d}^{-1}$ and $3.749 \mathrm{~d}^{-1}$; the latter of which agrees well with the orbital frequency derived from the photometry and from the radial velocity variations. The equivalent width measurements folded over Eq. (1) are shown in Fig. 6 (second panel from top). As expected for an irradiation effect, maximum equivalent width takes place at $\varphi \simeq 0.5$.
Table 2. The average radial velocities of the Balmer emission lines and $\mathrm{H} \beta$ equivalent widths of HS $1857+5144$ measured from the McDonald and WHT spectra.

\begin{tabular}{cccccc}
\hline \hline HJD 245 & $\begin{array}{c}V \\
\left(\mathrm{~km} \mathrm{~s}^{-1}\right)\end{array}$ & $\begin{array}{c}E W \\
(\AA)\end{array}$ & HJD 245 & $\begin{array}{c}V \\
\left(\mathrm{~km} \mathrm{~s}^{-1}\right)\end{array}$ & $\begin{array}{c}E W \\
(\AA)\end{array}$ \\
\hline 3202.6780 & $4.5 \pm 3.9$ & 10.8 & 3202.8808 & $157.4 \pm 6.7$ & 4.8 \\
3202.6857 & $-36.2 \pm 3.4$ & 9.1 & 3202.8884 & $161.1 \pm 6.2$ & 5.6 \\
3202.6954 & $-64.0 \pm 3.6$ & 9.7 & 3202.8954 & $161.8 \pm 6.4$ & 6.2 \\
3202.7037 & $-103.4 \pm 3.5$ & 8.4 & 3202.9058 & $140.2 \pm 5.5$ & 7.0 \\
3202.7134 & $-128.7 \pm 4.1$ & 8.2 & 3202.9134 & $115.5 \pm 5.3$ & 8.3 \\
3202.7211 & $-150.9 \pm 5.1$ & 8.2 & 3202.9204 & $85.9 \pm 4.8$ & 8.1 \\
3202.7287 & $-204.6 \pm 6.8$ & 7.7 & 3202.9294 & $58.8 \pm 4.6$ & 7.9 \\
3202.7405 & $-205.2 \pm 6.1$ & 5.0 & 3202.9371 & $28.6 \pm 4.5$ & 10.0 \\
3202.7482 & $-218.7 \pm 6.1$ & 4.4 & 3202.9447 & $-31.9 \pm 6.4$ & 6.8 \\
3202.7836 & - & -0.6 & 3202.9537 & $-44.8 \pm 4.7$ & 7.9 \\
3202.7912 & - & -2.3 & 3202.9614 & $-82.5 \pm 5.0$ & 9.6 \\
3202.8013 & - & -3.0 & 3205.6857 & $-224.3 \pm 8.1$ & 1.5 \\
3202.8114 & - & -3.3 & 3205.7468 & - & -1.9 \\
3202.8197 & - & -2.9 & 3205.8114 & $217.3 \pm 10.4$ & 1.4 \\
3202.8273 & - & -3.0 & 3205.8760 & $28.6 \pm 4.7$ & 9.5 \\
3202.8405 & - & -0.2 & 3919.3887 & $-57.8 \pm 2.8$ & 8.7 \\
3202.8482 & - & -1.0 & 3920.3912 & $155.6 \pm 3.5$ & 4.6 \\
3202.8572 & - & 0.1 & 3921.3888 & - & -2.7 \\
3202.8648 & $153.1 \pm 10.3$ & 1.2 & 3922.3880 & $-226.1 \pm 4.7$ & 2.1 \\
3202.8725 & $140.8 \pm 8.7$ & 2.5 & & & \\
\hline
\end{tabular}

Notes: The statistical error of the $\mathrm{H} \beta$ equivalent widths is $\sim 0.01-0.05 \AA$, which is negligible. The systematic error, however, is of the order $\sim 1 \AA$, depending on the details of how the continuum flux is determined.

\subsection{Photometric and spectroscopic phase relations}

The assumption that the emission lines in HS $1857+5144$ originate on the inner hemisphere of the secondary star as a result of strong irradiation from the hot primary star makes specific predictions on the relative phases of the photometric and spectroscopic variability. At superior conjunction of the secondary star when the irradiated side faces the observer, $\varphi=0.5$, the system appears brightest; the radial velocity of the secondary star is zero and crossing from red-shifted to blue-shifted velocities; the emission-line strength is at the maximum, and vice versa for the inferior conjunction of the secondary star at phase zero. Hence, one would expect an agreement in phase between the light curve and the equivalent width variation, and a 0.25 phase shift between those two parameters and the radial velocity curve (e.g. Thorstensen et al. 1978, 1994, 1996; Vennes \& Thorstensen 1996; Orosz et al. 1999; Hillwig et al. 2000; Kawka et al. 2002). Figure 6 shows the average radial velocities of the Balmer lines and the equivalent widths of $\mathrm{H} \beta$ as well as the $B$-band and $R$ band light curves folded over Eq. (1). The phase offset between the $B$-band and $R$-band light curves with respect to the equivalent width variation, as determined from sine fits, is $\sim 0.004$ for the $R$-band and $\sim 0.018$ for the $B$-band. The larger phase offset of the $B$-band light curve is probably related to the fact that it does not cover the entire orbital cycle, and hence the sine fit results in larger uncertainties. The phase of the folded equivalent width variation lags that of the radial velocity curve by $0.25 \pm 0.01$ orbital cycle.

The phase-dependent behaviour of the emission lines, and the relative phases of the photometric, radial velocity, and equivalent width variations found in our data corroborate the hypothesis of the emission lines in HS $1857+5144$ originating on the inner face of the secondary star illuminated by the hot white dwarf. 


\section{Stellar components}

\subsection{Light curve solution}

In order to determine additional constraints on the system parameters from the observed reflection effect we modelled the light curves of HS 1857+5144 with the "PHysics Of Eclipsing BinariEs" program PHOEBE ${ }^{3}$ (Prša \& Zwitter 2005), which is built on top of the widely used WD code (Wilson \& Devinney 1971; Wilson 1979, 1990). We simultaneously fitted the $R$-band and $B$-band data obtained at Kryoneri Observatory under the following assumptions: (a) circular orbits and synchronous rotation of the secondary star; (b) stellar surface temperature and brightness were computed assuming blackbody emission; (c) a detailed calculation of the reflection effect was adopted; (d) linear limb darkening was chosen, where the limb darkening coefficient was interpolated from Claret (2000); (e) gravity darkening exponents of 1 (von Zeipel 1924) and 0.32 (Lucy 1967) were used for radiative and convective stars, respectively; (f) no contribution fluxes from a spot or third light were applied.

In our analysis we tested a wide range of white dwarf masses, covering $M_{\mathrm{wd}}=0.3-1.4 M_{\odot}$. We then assumed an M-type companion star, testing the whole range of spectral types M9-M0 V, corresponding to masses, radii, and temperatures of $M_{\mathrm{sec}} \simeq 0.07-0.53 M_{\odot}, R_{\mathrm{sec}} \simeq 0.11-0.56 R_{\odot}$, and $T_{\mathrm{sec}} \simeq 2300-3800 \mathrm{~K}$, interpolated from Rebassa-Mansergas et al. (in preparation). Earlier spectral types than M0 V would imply extremely massive white dwarfs (Sect. 4.3) which are excluded by the spectral fit (Sect. 4.2) ${ }^{4}$. This approach allows us to search for possible solutions over a large range of possible mass ratios, $q=M_{\mathrm{sec}} / M_{\mathrm{wd}} \simeq 0.05-1.77$. For each input $q$ we fixed $P_{\text {orb }}=0.26633 \mathrm{~d}$ and $T_{\text {sec }}$ according to the selected spectral type of the companion star. The following parameters were free in the fits: $q$, white dwarf temperature $\left(T_{\mathrm{wd}}\right)$, orbital inclination $(i)$, surface potentials for both components, and albedo of the secondary star $(A L B 2)$. The fits for early type companions, M2-M0 V, do not reproduce well the observed $B$ and $R$ light curves, which supports the exclusion of early-type donors outlined above. We found that, independently from the details of a given fit, the system must contain a hot white dwarf with $T_{\text {wd }}>60000 \mathrm{~K}$ and a cool component with an albedo higher than that of a normal $\mathrm{M} \operatorname{star}(A L B 2>0.5)$ to reproduce the large amplitude observed in the light curves. Such high $T_{\mathrm{wd}}$ is also confirmed by the spectral fit to the WHT faint-phase spectrum in the following section. Fitting the $B$-band and $R$-band light curves alone provides a fairly large range of possible system parameters, $M_{\mathrm{wd}} \simeq 0.3-1.4 M_{\odot}, M_{\mathrm{sec}} \simeq 0.066-0.367 M_{\odot}$, (spectral type M9-M3 V), $i \sim 40^{\circ}-60^{\circ}$, and $T_{\text {wd }} \sim 60000-100000 \mathrm{~K}$. For a given $M_{\mathrm{sec}}$, a more massive white dwarf requires a larger inclination and a higher $T_{\mathrm{wd}}$.

Figure 6 (bottom two panels) presents the corresponding $R$-band and $B$-band synthetic light curves from the program PHOEBE for $M_{\mathrm{wd}}=0.72 M_{\odot}, M_{\mathrm{sec}}=0.21 M_{\odot}, i=53^{\circ}$, and $T_{\mathrm{wd}}=100000 \mathrm{~K}$, along with the observed light curves folded over the ephemeris in Eq. (1). The choice of this particular set of parameter is detailed below in Sect. 4.4, but other fits in the parameter range given above fit the data equally well.

\footnotetext{
${ }^{3}$ http://phoebe.fiz.uni-lj.si/

4 A strict upper limit on the mass of the companion comes from the fact that it is not Roche-lobe filling. Using $\bar{\rho} \simeq 107 P_{\text {orb }}^{-2}(\mathrm{~h})$ (e.g. Eggleton 1983), with $\bar{\rho}$, the average density of the donor, and $P_{\text {orb }}($ h), the orbital period in hours for a Roche-lobe filling star, the maximum mass of a main-sequence companion in HS $1857+5144$ is $M_{\text {sec }}<0.72 M_{\odot}$, corresponding to a spectral type $\mathrm{K} 3 \mathrm{~V}$ or later.
}

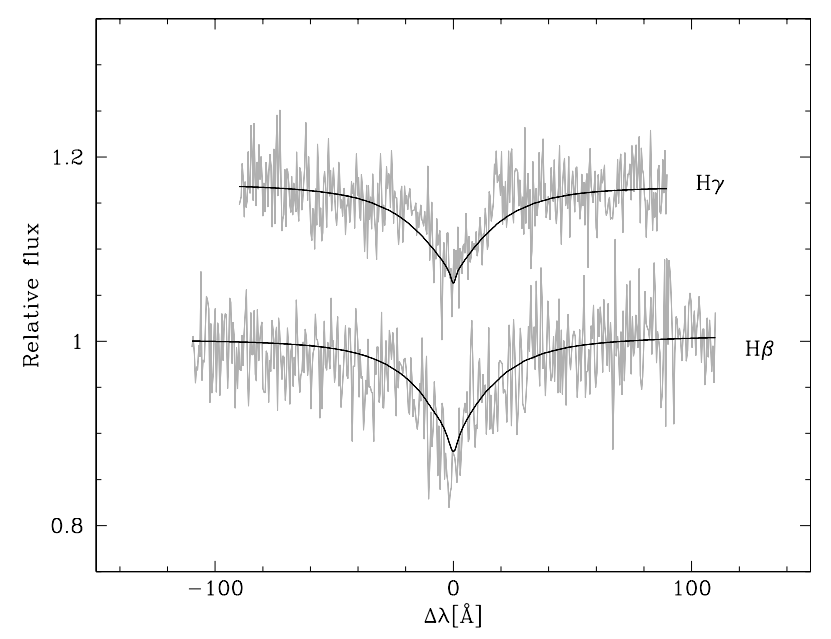

Fig. 7. $\mathrm{H} \beta$ and $\mathrm{H} \gamma$ from the WHT faint-phase spectrum $(\varphi=0.02)$ fitted with an LTE model for $T_{\mathrm{wd}}=100000 \mathrm{~K}$ and $\log g=8.0$.

\subsection{Spectral fit}

We performed a spectral fit to the faint-phase WHT spectrum of HS $1857+5144$ to obtain an independent estimate of $M_{\mathrm{wd}}$ and $T_{\mathrm{wd}}$, using both a grid of LTE pure-hydrogen models (Koester et al. 2005), and a grid of NLTE models with a variety of $\mathrm{He}$ abundances ${ }^{5}$. We fitted the $\mathrm{H} \beta$ and $\mathrm{H} \gamma$ absorption lines after normalising the continua of the observed data and the model spectra in the same way using a third-order polynomial. The fits suggest $80000 \mathrm{~K} \lesssim T_{\text {wd }} \lesssim 100000 \mathrm{~K}$ and $7.5 \lesssim \log g \lesssim 8.5$ (corresponding to $0.6 M_{\odot} \lesssim M_{\mathrm{wd}} \lesssim 1.0 M_{\odot}$ ) for the LTE models, and $70000 \mathrm{~K} \lesssim T_{\text {wd }} \lesssim 100000 \mathrm{~K}$ and $8.0 \lesssim \log g \lesssim 8.5$ (corresponding to $0.7 M_{\odot} \lesssim M_{\mathrm{wd}} \lesssim 1.0 M_{\odot}$ ) for the NLTE models. Figure 7 shows the best LTE fit to $\mathrm{H} \beta$ and $\mathrm{H} \gamma$ for $T_{\text {wd }}=100000 \mathrm{~K}$ and $\log g=8.0$. These numbers should be considered as rough estimates only, as the optical spectrum of HS $1857+5144$ is contaminated by flux from the companion star, which is very difficult to quantify. A more reliable temperature and mass estimate would require far-ultraviolet data, where the white dwarf dominates the emission of the system (Good et al. 2004). We conclude from this qualitative spectral analysis that the white dwarf in HS $1857+5144$ is indeed very hot, and given the high surface gravity preferred by the fits, is more likely to be a white dwarf than a subdwarf. The detection of He II $\lambda 4686$ then qualifies the primary as a DAO white dwarf.

\subsection{K-correction and mass ratio-inclination constraints}

As mentioned already in Sect.3.2, the emission lines in HS $1857+5144$ trace the orbit of the centre of light of the illuminated hemisphere of the secondary star, and not its centre of mass. Hence, for a dynamic assessment of the binary parameters, the measured velocity $K_{\mathrm{em}}$ (Sect. 3.2) has to be corrected accordingly. The radial velocity amplitude of the secondary star's centre of mass, $K_{\mathrm{sec} \text {, cor }}$ can be expressed according to Wade \& Horne (1988) as

$$
K_{\mathrm{sec}, \mathrm{cor}}=\frac{K_{\mathrm{em}}}{1-(1+q)(\Delta R / a)},
$$

where $\Delta R$ is the displacement of the centre of light from the centre of mass of the secondary star, with $0 \lesssim \Delta R \lesssim R_{\text {sec }}(\Delta R=0$ implies that the centre of light coincides with the centre of mass

\footnotetext{
${ }^{5}$ http://astro.uni-tuebingen.de/ rauch
} 
of the secondary star, whereas $\Delta R=R_{\mathrm{sec}}$ is the maximum possible displacement, where all the light comes from a small region on the secondary star closest to the primary star). Assuming that the emission due to irradiation is distributed uniformly over the inner hemisphere of the secondary star, and zero on its unirradiated face, $\Delta R=(4 / 3 \pi) R_{\text {sec }}$ (Wade \& Horne 1988; Wood et al. 1995; Orosz et al. 1999; Vennes et al. 1999). is

The expected radial velocity of the secondary $\operatorname{star}\left(K_{\mathrm{sec}, \mathrm{cal}}\right)$

$K_{\text {sec, cal }}=\frac{2 \pi a \sin i}{P_{\text {orb }}(1+q)}$

where $a$ is the binary separation. Equating $K_{\text {sec, cor }}=K_{\text {sec, cal }}$ then gives a unique $q$ for a given choice of $i$, Hence, a fixed value of $i$ projects onto a one-dimensional curve within the $\left(M_{\mathrm{wd}}, M_{\mathrm{sec}}\right)$ plane, and for the possible range of parameters considered here, those curves are nearly straight lines.

\subsection{Combined constraints}

In Sect. 4.1-4.3 above we have outlined what type of constraints on the system parameters of HS $1857+5144$ can be derived from the observed light curves, radial velocity variations, and the spectrum of the primary star. Here, we will combine all those independent constraints.

In a first step, we imposed a range of $i=40^{\circ}-60^{\circ}$, as suggested by the set of PHOEBE fits to the $B$-band and $R$-band light curves, on the combinations of $\left(M_{\mathrm{wd}}, M_{\mathrm{sec}}\right)$ which are consistent with the corrected radial velocity $K_{\text {sec, cor }}$ of the secondary star. The resulting parameter range is indicated by the gray shaded area in Fig. 8. In a second step, we inspected the individual light curve fits from the grid of PHOEBE runs, and required the inclination of a model for a given $\left(M_{\mathrm{wd}}, M_{\mathrm{sec}}\right)$ to fall within $\pm 5^{\circ}$ of the corresponding inclination constraint from the radial velocity of the secondary star. We introduced this "fuzziness" in inclination as a measure to account for systematic uncertainties within the $K$-correction and the light curve fits. Possible combinations of $\left(M_{\mathrm{wd}}, M_{\mathrm{sec}}\right)$ are indicated by filled circles in Fig. 8 , and trace a somewhat narrower band than the initial $i=40^{\circ}-60^{\circ}$ constraint. A final constraint comes from the spectral fit of the WHT faint phase spectrum (Sect. 4.2), which implied 0.6 $M_{\odot} \lesssim$ $M_{\mathrm{wd}} \lesssim 1.0 M_{\odot}$, shown as vertical dashed lines in Fig. 8 .

The combination of all constraints suggests $0.15 M_{\odot} \lesssim$ $M_{\text {sec }} \lesssim 0.30 M_{\odot}$ (spectral type M6-M4 V), 0.6 $M_{\odot} \lesssim M_{\mathrm{wd}} \lesssim$ $1.0 M_{\odot}, 70000 \mathrm{~K} \lesssim T_{\text {wd }} \lesssim 100000 \mathrm{~K}$, and $45^{\circ} \lesssim i \lesssim 55^{\circ}$. A substantial improvement on this set of parameters will require measuring the radial velocity amplitude of the white dwarf, $K_{\mathrm{wd}}$, and determining an accurate temperature for the primary. Both types of measurements could be easily obtained from time-resolved ultraviolet spectroscopy.

\subsection{MASS magnitudes}

HS $1857+5144$ is detected in the 2MASS (Skrutskie et al. 2006) at $J=15.09 \pm 0.04, H=15.05 \pm 0.08, K_{\mathrm{s}}=14.76 \pm 0.14$. Our ephemeris is good enough to establish the orbital phase of the 2MASS data, which is very close to orbital maximum, $\varphi \simeq 0.5$. In order to test to what extent the optical-infrared spectral energy distribution (SED) of HS $1857+5144$ is compatible with the stellar photometry and spectroscopy, we modelled the $B R J H K_{\mathrm{s}}$ parameter range obtained from the analysis of the time-resolved magnitudes with the sum of a white dwarf spectrum from Koester et al. (2005) and a blackbody representing the

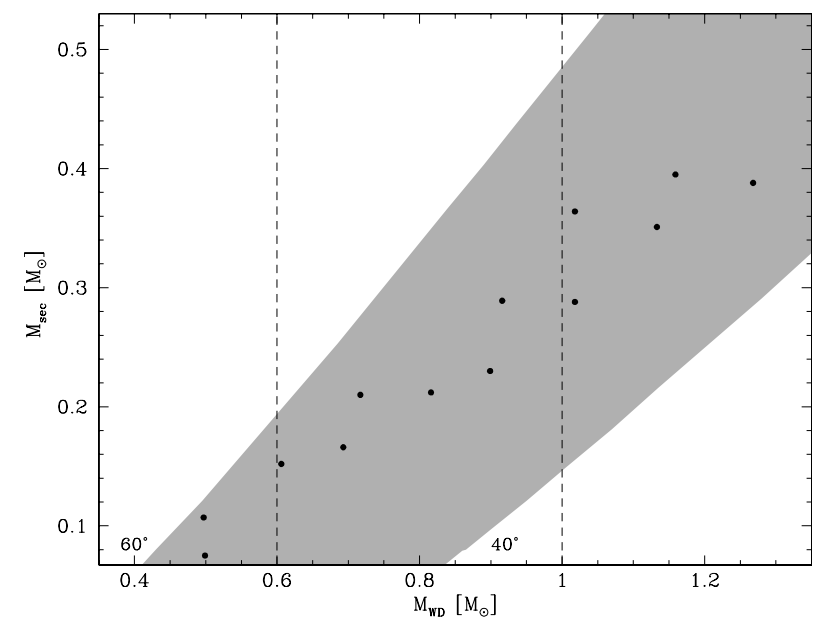

Fig. 8. Photometric and spectroscopic constraints on $\left(M_{\mathrm{wd}}, M_{\mathrm{sec}}\right)$. Gray shade area represents possible dynamical solutions from the "K-corrected" radial velocity of the secondary star for $K_{\mathrm{em}}=$ $185 \mathrm{~km} \mathrm{~s}^{-1}$ and for $i=40^{\circ}-60^{\circ}$. Filled circles represent possible solutions from the light curve analysis for given $\left(M_{\mathrm{wd}}, M_{\mathrm{sec}}\right)$ in which $i$ agrees with spectroscopic constraint within $\pm 5^{\circ}$. Dashed lines are upper and lower limits on $M_{\mathrm{wd}}$ derived from the spectral fit (see Sect. 4.4 for details).



Fig. 9. The $B R J H K_{\mathrm{s}}$ fluxes of HS $1857+5144$ (filled triangles) at orbital maximum $(\varphi \simeq 0.5)$ along with an example of a plausible fit (solid line) with the sum of a white dwarf spectrum (dots) and a blackbody (dashed line) representing the heated side of the low-mass companion, assuming $T_{\mathrm{wd}}=70000 \mathrm{~K}, T_{\mathrm{sec}}=6000 \mathrm{~K}, R_{\mathrm{wd}}=1.3 \times 10^{9} \mathrm{~cm}$, and $R_{\mathrm{sec}}=1.3 \times 10^{10} \mathrm{~cm}$, at a distance of $460 \mathrm{pc}$ (see Sect. 4.5 for details).

contribution of a (heated) low-mass companion. The data leave some freedom in the exact parameters, however, as an example, a fairly good fit is achieved for $T_{\mathrm{wd}}=70000 \mathrm{~K}, T_{\mathrm{sec}}=6000 \mathrm{~K}$, $R_{\mathrm{wd}}=1.3 \times 10^{9} \mathrm{~cm}$, corresponding to $M_{\mathrm{wd}} \simeq 0.6 M_{\odot}$, and $R_{\mathrm{sec}}=1.3 \times 10^{10} \mathrm{~cm}$, corresponding to spectral type of M6, at a distance of $460 \mathrm{pc}$ (Fig. 9). This solution coincides well with the photometric and spectroscopic constraints in Fig. 8. Overall our simple model confirms the stellar parameters established in the previous sections, with a slight preference for a white dwarf mass in the range $M_{\mathrm{wd}}=0.6-0.8 M_{\odot}$, and a companion star with a radius near the lower end of the determined range, i.e. corresponding to a spectral type M6-M5, and a distance to the system of $290-460 \mathrm{pc}$. 
Table 3. PCEBs with a large reflection effect.

\begin{tabular}{|c|c|c|c|c|c|c|c|c|c|c|}
\hline \multirow[t]{2}{*}{ Object } & \multirow{2}{*}{$\begin{array}{l}P_{\text {orb }} \\
\text { [d] }\end{array}$} & \multirow[t]{2}{*}{ SP1 } & \multirow[t]{2}{*}{ SPsec } & \multirow{2}{*}{$\begin{array}{c}T_{1} \\
{[\mathrm{~K}]} \\
\end{array}$} & \multirow{2}{*}{$\begin{array}{c}M_{1} \\
{\left[M_{\odot}\right]}\end{array}$} & \multirow{2}{*}{$\begin{array}{c}M_{\mathrm{sec}} \\
{\left[M_{\odot}\right]}\end{array}$} & \multicolumn{3}{|c|}{ Reflection effect [mag] } & \multirow[t]{2}{*}{ Ref. } \\
\hline & & & & & & & $B$ & $V$ & $R$ & \\
\hline NNSer & 0.130 & DAO & $\mathrm{M} 4.75 \mathrm{~V}$ & $57000 \pm 3000$ & $0.54 \pm 0.05$ & $0.150 \pm 0.008$ & 0.33 & 0.49 & 0.772 & 1,2 \\
\hline HS $1857+5144$ & 0.266 & DAO & M6-M4 V & $\sim 70000-100000$ & $\sim 0.6-1.0$ & $\sim 0.15-0.30$ & 0.7 & & 1.1 & 3 \\
\hline TW Crv & 0.328 & $\mathrm{sdO}$ & MV & $105000 \pm 20000$ & $\sim 0.55-0.61$ & $<0.3$ & 0.74 & 0.85 & 0.93 & 4,5 \\
\hline KV Vel & 0.357 & $\mathrm{sdO}, \mathrm{PN}$ & M V & $77000 \pm 3000$ & $0.63 \pm 0.03$ & $0.23 \pm 0.01$ & 0.49 & 0.55 & 0.61 & 6 \\
\hline V477 Lyr & 0.472 & sdOB, PN & & $60000 \pm 10000$ & $0.51 \pm 0.07$ & $0.15 \pm 0.02$ & 0.5 & 0.6 & & 7,8 \\
\hline V664 Cas & 0.582 & $\mathrm{sdO}, \mathrm{PN}$ & K5-F5 V & $83000 \pm 6000$ & & & & 1.15 & & 9,5 \\
\hline VW Pyx & 0.676 & $\mathrm{sdO}, \mathrm{PN}$ & & $85000 \pm 6000$ & & & & 1.36 & & 10,11 \\
\hline Abell 65 & 1 & sd?, PN & & $\sim 80000$ & & & & $>0.5$ & & 12,13 \\
\hline BE UMa & 2.291 & $\mathrm{sdO} / \mathrm{DAO}, \mathrm{PN}$ & K4-3 V & $105000 \pm 5000$ & $0.70 \pm 0.07$ & $0.36 \pm 0.07$ & & $\sim 1.3$ & & $14,15,16$ \\
\hline
\end{tabular}

References: (1) Haefner (1989); (2) Haefner et al. (2004); (3) this work; (4) Chen et al. (1995); (5) Exter et al. (2005); (6) Hilditch et al. (1996); (7) bond+grauer-87; (8) Pollacco \& Bell (1994); (9) shimanskiietal04-1; (10) Kohoutek \& Schnur (1982); (11) Exter et al. (2003); (12) Bond \& Livio (1990); (13) Walsh \& Walton (1996); (14) Ferguson et al. (1987); (15) Wood et al. (1995); (16) Ferguson et al. (1999).

\section{Discussion}

The analysis presented in Sect. 4.2 suggests that HS $1857+5144$ contains a hot white dwarf with $T_{\mathrm{wd}} \simeq 70000-100000 \mathrm{~K}$. The implied cooling age of the white dwarf is $1.2-6 \times 10^{5} \mathrm{yr}$ (Bergeron et al. 1995, and Bergeron 2002, private communication), making HS $1857+5144$ one of the youngest PCEBs known to date. Following the prescription of Schreiber \& Gänsicke (2003), and assuming the range of system parameters established in Sect. 4 as well as "classical" magnetic braking for the angular momentum loss mechanism, we estimate the period at which HS $1857+5144$ left the common envelope phase to be $P_{\mathrm{CE}} \simeq$ $0.266334-0.266345 \mathrm{~d}$, very close to its present orbital period. HS $1857+5144$ will evolve within the next $\sim 0.4-1.3 \times 10^{10} \mathrm{yr}$ into a semidetached $\mathrm{CV}$ configuration, and start mass transfer at an orbital period of $\simeq 0.08-0.13 \mathrm{~d}$, i.e. within or below the period gap. The large uncertainties on the future evolution are a consequence of the limited constraints on the system parameters. Additional systematic uncertainties in the actual strength of angular momentum loss from the orbit have not been taken into account.

Among 40 previously known PCEBs listed in Schreiber \& Gänsicke (2003), and Shimansky et al. (2006), only 8 systems display a reflection effect comparable to that of HS $1857+5144$ (Table 3). All those systems contain extremely hot white dwarfs or subdwarfs, and all are very young PCEBs that may serve as observational probes into our understanding of common envelope evolution.

A large reflection effect is expected for those PCEBs containing a hot subdwarf, because of the larger luminosity compared to a white dwarf of the same temperature, and indeed the majority of known PCEBs with a large reflection effect have sdO primary stars. So far only one PCEB, HS 1136+6646, containing a hot white dwarf similar to HS 1857+5144 (Sing et al. 2004) is known. The secondary star in HS $1136+6646$ has been suggested to be a K7-4 V star on the basis of its spectral type, which appears too early for the estimated mass of $0.34 M_{\odot}$. However, Sing et al. (2004) discuss the possibility that the secondary is overluminous as it is still out of thermal equilibrium after accreting at a high rate during the common envelope phase. The amplitude of the reflection effect in HS $1136+6646$ is much lower than in HS $1857+5144$, which is consistent with its longer orbital period of $0.84 \mathrm{~d}$. The other system most similar to HS $1857+5411$ is BE UMa, which has been classified as a borderline object between an sdO subdwarf and a DAO white dwarf (Liebert et al. 1995; Ferguson et al. 1999), and is associated with a planetary nebula. It is interesting to note that six out of the nine systems listed in Table 3 are still embedded in a planetary nebula. Our long-slit spectroscopy of HS $1857+5144$ does not reveal any noticeable trace of extended emission around $\mathrm{H} \alpha$, though a deep $\mathrm{H} \alpha$ image testing for remnant nebular emission would be useful. Similarly, no sign of extended $\mathrm{H} \alpha$ emission around HS $1136+6646$ has been observed. While the majority of very young PCEBs are still embedded in their planetary nebulae/common envelopes, the discovery of HS $1857+5144$ and HS $1136+6646$ suggests that the envelope can be dispersed rather quickly.

\section{Conclusions}

We have identified a pre-CV, HS $1857+5144$, containing a hot DAO white dwarf and a low mass M V star with an orbital period of $P_{\text {orb }}=383.52 \mathrm{~min}$. The light curves of HS $1857+5144$ display a very large reflection effect with peak-to-peak amplitude of 0.7 and $1.1 \mathrm{mag}$ in the $B$-band and $R$-band, respectively. Combining the results of our spectroscopic and photometric analysis, we constrain the system parameter to $0.15 M_{\odot} \lesssim M_{\text {sec }} \lesssim 0.30 M_{\odot}$ (spectral type M6-M4 V), $0.6 M_{\odot} \lesssim M_{\text {wd }} \lesssim 1.0 M_{\odot}, 70000 \mathrm{~K} \lesssim$ $T_{\text {wd }} \lesssim 100000 \mathrm{~K}$, and $45^{\circ} \lesssim i \lesssim 55^{\circ}$. Taking these parameters at face value HS $1857+5144$ is one of the youngest PCEBs known so far and has just emerged from the post common envelope phase. The system will eventually evolve into a cataclysmic variable, and start mass transfer within or below the 2-3 h orbital period gap.

Acknowledgements. A.A. thanks the Royal Thai Government for a studentship. B.T.G. and P.R.G. were supported by a PPARC Advanced Fellowship and a PDRA grant, respectively. The HQS was supported by the Deutsche Forschungsgemeinschaft through grants Re 353/11 and Re 353/22. We thank John Southworth for reducing the WHT spectra. Tom Marsh is acknowledged for developing and sharing his reduction and analysis package MOLLY. We thank the anonymous referee for his/her comments that lead to an improved presentation of the paper. This publication makes use of data products from the Two Micron All Sky Survey, which is a joint project of the University of Massachusetts and the Infrared Processing and Analysis Center/California Institute of Technology, funded by the National Aeronautics and Space Administration and the National Science Foundation.

Based in part on observations collected at the Centro Astronómico Hispano Alemán (CAHA) at Calar Alto, operated jointly by the Max-Planck Institut für Astronomie and the Instituto de Astrofísica de Andalucía (CSIC); on observations made with the $2.7 \mathrm{~m}$ telescope at the McDonald Observatory of the University of Texas at Austin (Texas); on observations made at the $1.2 \mathrm{~m}$ telescope, located at Kryoneri Korinthias, and owned by the National Observatory of Athens, Greece; on observations made with the William Herschel Telescope, operated on the island of La Palma by the Instituto de Astrofísica de Canarias (IAC) at the Spanish Observatorio del Roque de los Muchachos; on observations 
made with the IAC80 telescope, operated on the island of Tenerife by the IAC at the Spanish Observatorio del Teide of the IAC; on observations made with the Optical Groud Station telescope, operated on the island of Tenerife by the European Space Agency, in the Spanish Observatorio del Teide of the IAC.

\section{References}

Aungwerojwit, A., Gänsicke, B. T., Rodríguez-Gil, P., et al. 2005, A\&A, 443, 995

Barman, T. S., Hauschildt, P. H., \& Allard, F. 2004, ApJ, 614, 338

Bergeron, P., Wesemael, F., \& Beauchamp, A. 1995, PASP, 107, 1047

Bertin, E., \& Arnouts, S. 1996, A\&AS, 117, 393

Bond, H. E., \& Livio, M. 1990, ApJ, 355, 568

Chen, A., O'Donoghue, D., Stobie, R. S., et al. 1995, MNRAS, 275, 100

Claret, A. 2000, A\&A, 363, 1081

Eggleton, P. P. 1983, ApJ, 268, 368

Exter, K. M., Pollacco, D. L., \& Bell, S. A. 2003, MNRAS, 341, 1349

Exter, K. M., Pollacco, D. L., Maxted, P. F. L., Napiwotzki, R., \& Bell, S. A. 2005, MNRAS, 359, 315

Ferguson, D. H., \& James, T. A. 1994, ApJS, 94, 723

Ferguson, D. H., Liebert, J., Cutri, R., et al. 1987, ApJ, 316, 399

Ferguson, D. H., Liebert, J., Haas, S., Napiwotzki, R., \& James, T. A. 1999, ApJ, 518,866

Ferguson, D. H., McGraw, J. T., Spinrad, H., Liebert, J., \& Green, R. F. 1981, ApJ, 251, 205

Gänsicke, B. T., Hagen, H. J., \& Engels, D. 2002, in The Physics of Cataclysmic Variables and Related Objects, ed. B. T. Gänsicke, K. Beuermann, \& K. Reinsch, ASP Conf. Ser., 261, 190

Gänsicke, B. T., Araujo-Betancor, S., Hagen, H.-J., et al. 2004, A\&A, 418, 265

Good, S. A., Barstow, M. A., Holberg, J. B., et al. 2004, MNRAS, 355, 1031

Haefner, R. 1989, A\&A, 213, L15

Haefner, R., Fiedler, A., Butler, K., \& Barwig, H. 2004, A\&A, 428, 181

Hagen, H.-J., Groote, D., Engels, D., \& Reimers, D. 1995, A\&AS, 111, 195
Heber, U., Drechsel, H., Østensen, R., et al. 2004, A\&A, 420, 251 Hilditch, R. W., Harries, T. J., \& Hill, G. 1996, MNRAS, 279, 1380

Hillwig, T. C., Honeycutt, R. K., \& Robertson, J. W. 2000, AJ, 120, 1113

Kawka, A., Vennes, S., Koch, R., \& Williams, A. 2002, AJ, 124, 2853

Koester, D., Napiwotzki, R., Voss, B., Homeier, D., \& Reimers, D. 2005, A\&A, 439,317

Kohoutek, L., \& Schnur, G. F. O. 1982, MNRAS, 201, 21

Liebert, J., Tweedy, R. W., Napiwotzki, R., \& Fulbright, M. S. 1995, ApJ, 441, 424

Lucy, L. B. 1967, Z. Astrophys., 65, 89

Orosz, J. A., Wade, R. A., Harlow, J. J. B., et al. 1999, AJ, 117, 1598

Pollacco, D. L., \& Bell, S. A. 1994, MNRAS, 267, 452

Prša, A., \& Zwitter, T. 2005, ApJ, 628, 426

Schreiber, M. R., \& Gänsicke, B. T. 2003, A\&A, 406, 305

Schwarzenberg-Czerny, A. 1996, ApJ, 460, L107

Shimansky, V., Sakhibullin, N. A., Bikmaev, I., et al. 2006, A\&A, 456, 1069

Sing, D. K., Holberg, J. B., Burleigh, M. R., et al. 2004, AJ, 127, 2936

Skrutskie, M. F., Cutri, R. M., Stiening, R., et al. 2006, AJ, 131, 1163

Thorstensen, J. R., Charles, P. A., Bowyer, S., \& Margon, B. 1978, ApJ, 223, 260

Thorstensen, J. R., Vennes, S., \& Shambrook, A. 1994, AJ, 108, 1924

Thorstensen, J. R., Vennes, S., \& Bowyer, S. 1996, ApJ, 457, 390

Vennes, S., \& Thorstensen, J. R. 1996, AJ, 112, 284

Vennes, S., Thorstensen, J. R., \& Polomski, E. F. 1999, ApJ, 523, 386

von Zeipel, H. 1924, MNRAS, 84, 665

Wade, R. A., \& Horne, K. 1988, ApJ, 324, 411

Walsh, J. R., \& Walton, N. A. 1996, A\&A, 315, 253

Willems, B., \& Kolb, U. 2004, A\&A, 419, 1057

Wilson, R. E. 1979, ApJ, 234, 1054

Wilson, R. E. 1990, ApJ, 356, 613

Wilson, R. E., \& Devinney, E. J. 1971, ApJ, 166, 605

Wood, J. H., Robinson, E. L., \& Zhang, E.-H. 1995, MNRAS, 277, 87

York, D. G., Adelman, J., Anderson, J. E., et al. 2000, AJ, 120, 1579 